\title{
Participants
}

Bagchi, Arunabha

Faculty of Mathematical Sciences University Twente, P.O.Box 217, 7500AE Enschede, The Netherlands

email: A.Bagchi@math.utwente.nl

Bielecki, Tomasz R.

Mathematics Department, The Northeastern Illinois University, 5500 North St. Louis Avenue, Chicago, IL 60625-4699, USA

email: T-Bielecki@neiu.edu

Chen, Zengjing

Department of Mathematics, Shandong University, Jinan 250100, China email: chzengjing@hotmail.com

Frittelli, Marco

Dipartimento Metodi Quantitativi, Facolte di Economia, Universite di Milano

- Bicocca, Piazza dell'Ateneo Nuovo 1, 20126, Milano, Italy

email: marco.frittelli@unimib.it

Guo, Xin

P.O. Box 218, IBM T. J. Watson Research Center, Yorktown Heights, NY 10598, USA

email: xinguo@us.ibm.com

Hu, Yaozhong

Department of Mathematics, University of Kansas, 405 Snow Hall, Lawrence, KS 66045-2142, USA

email: hu@math.ukans.edu

$\mathrm{Li}$, Xunjing

Department of Mathematics, Fundan University, Shanghai 200433, China email: $x j l i @ f u d a n . e d u . c n$

Liu, Hong

Olin School of Business, Washington University, St. Louis, MO 63130, USA email: LIUH@olin.wustl.edu

Ma, Jin

Department of Mathematics, Purdue University, West Lafayette, IN 47907, USA

email: majin@math.purdue.edu 
Nagai, Hideo

Department of Mathematical Science, Graduate School of Engineering Science, Osaka University, Toyonaka, 560-8531, Japan

email: nagai@sigmath.es.osaka-u.ac.jp

Peng, Shige

Department of Mathematics, Shandong University, Jinan 250100, China

email: peng@sdu.edu.cn

Platen, Eckhard

University of Technology Sydney, School of Finance \& Economics and Department of Mathematical Sciences, PO Box 123, Broadway, NSW, 2007, A ustralia email: eplaten@uts.edu.au

Pliska, Stanley

Department of Finance, University of Illinois at Chicago, 601 S. Morgan Street, Chicago, IL 60607-7124, USA

email: srpliska@uic.edu

Runggaldier, Wolfgang

Dipartimento di Matematica Pura ed Applicata, Università di Padova, 7 Via Belzoni, I - 35131Padova, Italy

email: runggal@math.unipd.it

Rutkowski, Marek

Faculty of Mathematics and Information Science, Politechnika Warszawska, pl. Politechniki 1, 00-661 Warszawa, Poland

email: markrut@mini.pw.edu.pl

Savine, Antoine

Asia Group, BNP-Paribas, 18th floor, Tokyo Sankei Building, 1-7-2 Otemachi, Chiyoda-Ku, Tokyo 100-0004, Japan

email: antoine.savine@japan.bnpparibas.com

Sondermann, D.

University of Bonn, Department of Economics, Adenauer Allee 24, 53113 Bonn, Germany

email: sonderma@finasto.uni-bonn.de

Stettner, Lukasz

Institute of Mathematics, Polish Academy of Sciences, Sniadeckich 8, 00-950

Warsaw, Poland

email: stettner@panim.impan.gov.pl 
Sun, Yeneng

Department of Mathematics, National University of Singapore, 2 Science Drive 2, Singapore 117543

email: matsuny@nus.edu.sg

Tang, Shanjian

Department of Mathematics, Fudan University, Shanghai 200433, China email: sjtang@online.sh.cn

Vorst, Ton

Econometric Institute, Erasmus University Rotterdam, P.O. Box 1738, 3000 DR Rotterdam, The Netherlands

email:vorst@few.eur.nl

Yan, Jia-an

Institute of Applied Mathematics, Academy of Mathematics and System Sciences, Chinese Academy of Sciences, Beijing 100080, China

email: jayan@amath7.amt.ac.cn

Yang, Hailiang

Department of Statistics and Actuarial Science, The University of Hong Kong, Pokfulam Road, Hong Kong, China

email: hlyang@hkusua.hku.hk

Yao, David

Department of Systems Engineering and Engineering Management, The Chinese University of Hong Kong, Hong Kong, China

email:yao@se.cuhk.edu.hk

Yin, George

Department of Mathematics, Wayne State University, Detroit, MI 48202, USA email: gyin@math.wayne.edu

Yong, Jiongmin

Department of Mathematics, Fundan University, Shanghai 200433, China

email: jyong@fudan.edu.cn

Zhang, Qing

Department of Mathematics, University of Georgia, Athens, GA 30602, USA

email: qingz@control.math.uga.edu

Zhang, Qiang

Department of Mathematics, and Department of Economics and Finance City University of Hong Kong, 83 Tat Chee Avenue, Kowloon, Hong Kong, China email: mazq@math.cityu.edu.hk 


\section{Zhang, Shuzhong}

Department of Systems Engineering and Engineering Management, The Chinese University of Hong Kong, Hong Kong, China

email: zhang@se.cuhk.edu.hk

\section{Zhou, Xunyu}

Department of Systems Engineering and Engineering Management, The Chinese University of Hong Kong, Hong Kong, China email: xyzhou@se.cuhk.edu.hk 